\title{
LOG-CONCAVITY RESULTS FOR A BIPARAMETRIC AND AN ELLIPTIC EXTENSION OF THE $q$-BINOMIAL COEFFICIENTS
}

\author{
MICHAEL J. SCHLOSSER, KOUSHIK SENAPATI, AND ALI K. UNCU \\ Dedicated to Bruce Berndt, on the occasion of his 80th birthday.
}

\begin{abstract}
We establish discrete and continuous log-concavity results for a biparametric extension of the $q$-numbers and of the $q$-binomial coefficients. By using classical results for the Jacobi theta function we are able to lift some of our log-concavity results to the elliptic setting. One of our main ingredients is a putatively new lemma involving a multiplicative analogue of Turán's inequality.
\end{abstract}

\section{INTRODUCTION}

In this paper we extend some well-known properties of the $q$-numbers and $q$-binomial coefficients (a.k.a. Gaußian binomial coefficients) to settings involving extra parameters. In particular, we establish discrete and continuous log-concavity results for certain uniparametric, biparametric, and even elliptic extensions of the $q$-numbers and $q$-binomial coefficients.

Given any complex $q \neq 1$, the $q$-analogue of a complex number $x$ is defined by

$$
[x]_{q}:=\frac{1-q^{x}}{1-q} .
$$

We refer to $[x]_{q}$ as a $q$-number (or basic number). The $q$-numbers play an important role in the theory of integer partitions (see Andrews' book [1] and historical references cited in there). One can recover $x$ from $[x]_{q}$ by letting $q$ tend to 1 . Log-concavity results for $q$-binomial coefficients were given by Butler [2], Krattenthaler [5] and Sagan [7]. (In this connection it should be mentioned that the $q$-log-concavity or weighted log-concavity considered in the literature implies log-concavity if the weights are non-negative.) Recently Kalmykov and Karp [4] established log-concavity results (or equivalently, Turán type inequalities) for specific basic hypergeometric series.

For $a, b, q \in \mathbb{C}$ we define the $a, b ; q$-extension of a complex number $x$ as follows:

$$
[x]_{a, b ; q}:=\frac{\left(1-q^{x}\right)\left(1-a q^{x}\right)(1-b q)(1-a q / b)}{(1-q)(1-a q)\left(1-b q^{x}\right)\left(1-a q^{x} / b\right)},
$$

2010 Mathematics Subject Classification. Primary 05A20; Secondary 05A10, 05A30, 11F27, 26D20, $33 \mathrm{E} 05$.

Key words and phrases. log-concavity, $q$-series, binomial coefficients, theta functions, elliptic functions, Turán's inequality. 
where the variables are chosen such that none of the denominator factors vanish. (This definition corresponds to that of the $a, b ; q$-numbers considered by the first author and Yoo in [11, 12, 13, 15]. In the latter $b$ has to be replaced by $b q^{-1}$ to match the definition used in (1.1).) Letting $a \rightarrow 0$ followed by $b \rightarrow 0$ (or $b \rightarrow 0$ followed by $a \rightarrow \infty$ ), the $a, b ; q$-numbers reduce to the basic numbers.

In Section 2 we list some elementary properties of the $a, b ; q$-numbers and explain the various notions of log-concavity we are concerned about in this paper. Section 3 deals with results about the log-concavity of the $a, b ; q$-numbers. The lemma proved in that section involves a multiplicative analogue of Turán's inequality and plays a key role in proving results involving $a, b ; q$-numbers and $a, b ; q$-binomial coefficients, and in proving results in the (more general) elliptic setting. Section 4 is devoted to log-concavity results for uni- and biparametric extensions of the $q$-binomial coefficient. The $a, b ; q$-numbers can be further extended to the elliptic numbers that appeared in [11, 12, 13, 15], and are the contents of study in Sections 5 and 6. Our elliptic numbers are indeed elliptic functions (i.e., they are meromorphic and doubly periodic); they are expressed as certain ratios of theta functions. Accordingly, the analysis in Sections 5 and 6 involves some machinery from the theory of Jacobi theta functions (or, equivalently, of the Weierstraß sigma function) which is classical but not so well-known in the community of $q$-series, which is the reason why we cover this material in separate sections of our paper. Finally, in Section 7 we conclude with an outlook of further open problems.

\section{Preliminaries}

It is a matter of simple algebra to verify for arbitrary $x$ and $y$ the following addition formula for the $a, b ; q$-numbers defined in (1.1):

$$
[x]_{a, b ; q}+W_{a, b ; q}(x)[y-x]_{a q^{2 x}, b q^{x} ; q}=[y]_{a, b ; q},
$$

where $W_{a, b ; q}(x)$ is the $a, b ; q$-weight, defined by

$$
W_{a, b ; q}(x)=\frac{\left(1-a q^{1+2 x}\right)(1-b)(1-b q)(1-a / b)(1-a q / b)}{(1-a q)\left(1-b q^{x}\right)\left(1-b q^{1+x}\right)\left(1-a q^{x} / b\right)\left(1-a q^{1+x} / b\right)} q^{x} .
$$

Now if we impose $0<q<1$ and $0<a<b<1$ (in particular, all variables are presumed to be real), it is easy to see that for any real $x>0$ we have

$$
[x]_{a, b ; q}>0 \quad \text { and } \quad W_{a, b ; q}(x)>0,
$$

as all the factors appearing in the respective quotients are manifestly positive.

It is also easy to observe the following three properties of the $a, b ; q$-numbers and the associated $a, b ; q$-weights:

$$
\begin{aligned}
& {[0]_{a, b ; q}=0 \quad \text { and } \quad W_{a, b ; q}(0)=1,} \\
& {[x]_{a, b ; q} \geq[y]_{a, b ; q} \quad \text { for } \quad 0<q<1, \quad x \geq y \geq 0, \quad \text { and } \quad 0<a<b<1} \\
& {[x]_{a, b ; q}=-W_{a, b ; q}(x)[-x]_{a q^{2 x}, b q^{x} ; q} .}
\end{aligned}
$$


The relation (2.3b) follows from the addition formula in (2.1), with $x$ and $y$ having been interchanged, as for $x>y>0$ the difference $[x]_{a, b ; q}-[y]_{a, b ; q}$ is $W_{a, b ; q}(y)[x-y]_{a q^{2 y}, b q^{y} ; q}$ which is positive by (2.2). While we could use the above relation (2.3c) to deal with the $a, b ; q$-numbers of negative argument, in this paper we shall restrict our attention to the case that the arguments are non-negative real numbers.

There are two intermediate extensions from the basic-numbers to the $a, b ; q$-numbers. These two intermediate extensions correspond to the limits $b \rightarrow 0$, and to $a \rightarrow 0$, in the $a, b$; $q$-numbers, respectively. Specifically, one can let $b \rightarrow 0$ (or $b \rightarrow \infty)$ in (1.1), by which one obtains the $a ; q$-numbers (studied in [12, 14, 15]):

$$
[x]_{a ; q}:=[x]_{a, 0 ; q}=\frac{\left(1-q^{x}\right)\left(1-a q^{x}\right)}{(1-q)(1-a q)} q^{1-x} .
$$

These do not only generalize the standard $q$-numbers $[x]_{q}$ obtained by letting $a \rightarrow 0$ in (2.4), but also the quantum numbers $\langle x\rangle_{q}:=\left(q^{x}-q^{-x}\right) /\left(q-q^{-1}\right)$ (which frequently appear in physical models), obtained by letting $a \rightarrow-1$ in (2.4).

One can also let $a \rightarrow 0$ (or $a \rightarrow \infty)$ in (1.1) and arrive at the $(b ; q)$-numbers

$$
[x]_{(b ; q)}:=[x]_{0, b ; q}=\frac{\left(1-q^{x}\right)(1-b q)}{(1-q)\left(1-b q^{x}\right)} .
$$

We decided to put parantheses in " $(b ; q)$-numbers" but none in " $a ; q$-numbers" to distinguish them in notation, thus to avoid confusion. (For instance, we have $[x]_{(0 ; q)}=[x]_{q}$ but $[x]_{0 ; q}=[x]_{q^{-1}}$.)

In terms of standard terminology for basic hypergeometric series (cf. [3]), the basic hypergeometric expression on the right-hand side of (2.4) is well-poised and that on the right-hand side of (2.5) is balanced. It should come to no surprise that the right-hand side of (1.1) is well-poised and balanced (while the corresponding expression for the weight in $(2.1 \mathrm{~b})$ is even very-well-poised and balanced).

We now explain different notions of log-concavity.

Definition 1. A sequence of real numbers $\left(a_{k}\right)_{k=0}^{\infty}$ (indexed by non-negative integers) is called log-concave if

$$
a_{k}^{2} \geq a_{k+1} a_{k-1}
$$

for all $k \geq 1$. Similarly, one calls a sequence $\left(a_{k}\right)_{k=0}^{\infty}$ strongly log-concave if

$$
a_{k} a_{l} \geq a_{k+1} a_{l-1}
$$

for all positive integers $k$ and $l$ with $k \geq l$.

It is clear that if the $a_{k}$ are all positive (or all negative), log-concavity implies strong log-concavity since (2.6) then is equivalent to

$$
\frac{a_{k}}{a_{k+1}} \geq \frac{a_{k-1}}{a_{k}},
$$

which can be iterated to establish (2.7). 
We will also use the notions of log-concavity and strong log-concavity in the continuous setting.

Definition 2. A function $a(x)$ depending on a non-negative real variable $x$ is called continuously log-concave if

$$
a(x)^{2} \geq a(x+r) a(x-r),
$$

for all $x \geq r \geq 0$, and continuously strongly log-concave if

$$
a(x) a(y) \geq a(x+r) a(y-r)
$$

for all real $x \geq y \geq r \geq 0$.

Again it is easy to see that if $a(x)>0$ for all $x \geq 0$ (or $a(x)<0$ for all $x \geq 0$ ) log-concavity implies strong log-concavity since the down-shift by $r$ of the arguments, $a(x) / a(x+r) \geq a(x-r) / a(x)$, can be iterated with an additional down-shift by $x-y$ to establish (2.9).

For a non-vanishing positive (or non-vanishing negative) function $a(x), x \geq 0$, one can equivalently express the continuous strong log-concavity as

$$
\frac{a(x+r) a(y-r)}{a(x) a(y)} \leq 1,
$$

where $x \geq y \geq r \geq 0$. Similar reformulations can be applied to the other notions of log-concavity considered above and we will be using them when convenient.

Example 3. Maybe the most trivial example of a continuously strong log-concave function is the identity on $[0, \infty)$. Indeed, assuming $x \geq y \geq r>0$ (the case $r=0$ of (2.9) is trivial),

$$
\frac{(x+r)(y-r)}{x y}<1
$$

of course holds, since $x y-(x+r)(y-r)=r(r+x-y)>0$. This simple fact is already responsible for the continuous strong log-concavity of the continuous binomial coefficients (which were recently studied by Salwinski [8] who proved identities satisfied by them, among them also a continuous binomial theorem), defined by

$$
\left(\begin{array}{l}
x \\
k
\end{array}\right)=\frac{\Gamma(1+x)}{\Gamma(1+k) \Gamma(1+x-k)} \quad \text { for } x, k \in \mathbb{C}, x \notin-1,-2, \ldots
$$

By virtue of Euler's product formula for the gamma function,

$$
\Gamma(1+x)=\prod_{j=1}^{\infty} \frac{j^{1-x}(1+j)^{x}}{x+j} \quad \text { for } x \in \mathbb{C}, x \notin-1,-2, \ldots,
$$

we may rewrite Equation (2.12) in the following convenient product form:

$$
\left(\begin{array}{l}
x \\
k
\end{array}\right)=\prod_{j=1}^{\infty} \frac{(k+j)(x-k+j)}{j(x+j)} \quad \text { for } x, k \in \mathbb{C}, x \notin-1,-2, \ldots
$$


It is now easy to deduce the following result: For any real $x, y, k, l, r$ satisfying $x \geq y$, $k \geq l \geq r \geq 0$, and $y-l \geq x-k$, we have the continuous strong log-concavity

$$
\left(\begin{array}{l}
x \\
k
\end{array}\right)\left(\begin{array}{l}
y \\
l
\end{array}\right) \geq\left(\begin{array}{c}
x \\
k+r
\end{array}\right)\left(\begin{array}{c}
y \\
l-r
\end{array}\right) \text {. }
$$

Proof. The $r=0$ case is trivial, so assume $r>0$. After canceling common factors we see that

$$
\frac{\left(\begin{array}{c}
x \\
k+r
\end{array}\right)\left(\begin{array}{c}
y \\
l-r
\end{array}\right)}{\left(\begin{array}{l}
x \\
k
\end{array}\right)\left(\begin{array}{l}
y \\
l
\end{array}\right)}=\prod_{j=1}^{\infty} \frac{(k+r+j)(x-k-r+j)(l-r+j)(y-l+r+j)}{(k+j)(x-k+j)(l+j)(y-l+j)}<1,
$$

since by taking different instances of (2.11), we have

$$
\frac{(k+r+j)(l-r+j)}{(k+j)(l+j)}<1, \quad \text { and } \quad \frac{(y-l+r+j)(x-k-r+j)}{(y-l+j)(x-k+j)}<1,
$$

for each $j \geq 1$, which establishes the claim.

\section{LOG-CONCAVITY OF $a, b ; q$-NUMBERS}

To deal with the continuous log-concavity of the $a ; q^{-}$, the $(b ; q)$ - and the $a, b ; q$-numbers we will make use of the following elementary result.

Proposition 4. For $0<q<1$ and $0 \leq \nu<1$ the function $x \mapsto\left(1-\nu q^{x}\right), x \geq 0$ is continuously strongly log-concave.

Proof. Assume that $x \geq y \geq r \geq 0$. Using the definition (2.9) directly and rearranging the terms yields

$$
\left(1-\nu q^{x}\right)\left(1-\nu q^{y}\right)-\left(1-\nu q^{x+r}\right)\left(1-\nu q^{y-r}\right)=\nu q^{y-r}\left(1-q^{r}\right)\left(1-q^{x-y+r}\right) \geq 0 .
$$

In particular, we can utilize Proposition 4 to establish the following result involving the a; q-numbers.

Theorem 5. For $0<q<1$ and $0<a<1$ the a; q-numbers $[x]_{a ; q}$ are continuously strongly log-concave.

Proof. Let $x \geq y \geq r \geq 0$. Using (2.10) we have to show that the fraction

$$
\frac{[x+r]_{a ; q}[y-r]_{a ; q}}{[x]_{a ; q}[y]_{a ; q}}=\frac{\left(1-q^{x+r}\right)\left(1-q^{y-r}\right)}{\left(1-q^{x}\right)\left(1-q^{y}\right)} \frac{\left(1-a q^{x+r}\right)\left(1-a q^{y-r}\right)}{\left(1-a q^{x}\right)\left(1-a q^{y}\right)}
$$

is less or equal to 1 . Now the two fractions on the right-hand side are both non-negative and less than or equal to 1 by virtue of Proposition 4 (with $\nu=1$ and $\nu=a$ ), and (2.10). Thus their product is less than or equal to 1.

The same argument is not applicable to the $(b ; q)$-numbers $[x]_{(b ; q)}$, nevertheless we can show that the $(b ; q)$-numbers possess the continuously strong log-concavity property by elementary algebra. 
Theorem 6. For $0<q<1$ and $0<b<1$ the $(b ; q)$-numbers $[x]_{(b ; q)}$ are continuously strongly log-concave.

Proof. Let $x \geq y \geq r \geq 0$. By direct computation, we see that

$$
\begin{aligned}
& {[x]_{(b ; q)}[y]_{(b ; q)}-[x+r]_{(b ; q)}[y-r]_{(b ; q)}} \\
& =\frac{\left(1-q^{r}\right)\left(1-q^{x-y+r}\right)(1-b)(1-b q)^{2}\left(1-b q^{x+y}\right)}{(1-q)^{2}\left(1-b q^{x}\right)\left(1-b q^{x+r}\right)\left(1-b q^{y}\right)\left(1-b q^{y-r}\right)} q^{y-r} .
\end{aligned}
$$

All the factors appearing in the fraction on the right-hand side are non-negative. Thus the whole product is non-negative, so (2.9) holds.

We now move our attention to the $a, b ; q$-numbers. Before we show the continuous strong log-concavity of these numbers according to Definition 2, we show that these numbers satisfy an $a, b ; q$-version of continuous strong log-concavity where certain shifts of the variables $a$ and $b$ occur.

Theorem 7. For all real $x, y, a, b, q$ satisfying $0<q<1,0<a<b<1$, and $x \geq y \geq$ $r \geq 0$, the $a, b ; q$-numbers $[x]_{a, b ; q}$ satisfy the inequality

$$
[x]_{a q^{2 r}, b q^{r} ; q}[y]_{a, b ; q} \geq[x+r]_{a, b ; q}[y-r]_{a q^{2 r}, b q^{r} ; q} .
$$

Proof. Using (2.1), we have

$$
\begin{aligned}
& {[x]_{a q^{2 r}, b q^{r} ; q}[y]_{a, b ; q}-[x+r]_{a, b ; q}[y-r]_{a q^{2 r}, b q^{r} ; q}} \\
& =[x]_{a q^{2 r}, b q^{r} ; q}\left([r]_{a, b ; q}+W_{a, b ; q}(r)[y-r]_{a q^{2 r}, b q^{r} ; q}\right) \\
& \quad-\left([r]_{a, b ; q}+W_{a, b ; q}(r)[x]_{a q^{2 r}, b q^{r} ; q}\right)[y-r]_{a q^{2 r}, b q^{r} ; q} \\
& \quad=[r]_{a, b ; q}\left([x]_{a q^{2 r}, b q^{r} ; q}-[y-r]_{a q^{2 r}, b q^{r} ; q}\right) \\
& =[r]_{a, b ; q} W_{a q^{2 r}, b q^{r} ; q}(y-r)[x-y+r]_{a q^{2 y}, b q^{y} ; q} .
\end{aligned}
$$

The non-negativity of the last expression is clear from (2.2).

We can actually avoid using shifts of the variables $a$ and $b$ and show (pure) continuous strong log-concavity of the $a, b ; q$-numbers.

Theorem 8. The $a, b ; q$-numbers $[x]_{a, b ; q}$ are continuously strongly log-concave. In particular, for all real $x, y, a, b, q$ satisfying $0<q<1,0<a<b<1$, and $x \geq y \geq r \geq 0$, we have

$$
[x]_{a, b ; q}[y]_{a, b ; q} \geq[x+r]_{a, b ; q}[y-r]_{a, b ; q} .
$$

Theorem 8 is a direct consequence of a general lemma, see Lemma 9 below, which involves a multiplicative analogue of Turán's inequality. In its formulation, the role of the variable $\delta$, which is assumed to be a fixed non-negative real number, at first may seem to be enigmatic. Indeed, for the sole purpose of proving Theorem 8 , the case $\delta=0$ would completely suffice. However, we shall also employ the lemma when dealing with theta functions in the proofs of Theorems 14 and 15 and there we require $\delta$ to be a specific positive real number. 
Lemma 9. Let $0 \leq \delta<\lambda$. Let $f$ be a continuous positive real function on the interval $[\delta, \lambda]$, twice differentiable with values of first and second derivatives being negative on the interval $(\delta, \lambda)$. Let $\delta<a \leq b<\lambda$. Then we have the inequality

$$
\frac{f(\lambda) f(a)}{f(b) f(\lambda a / b)} \leq 1
$$

Proof. Without loss of generality, assume that $b \geq \lambda a / b$; if not, one can switch $b$ and $\lambda a / b$. Let $x=f(\lambda a / b)-f(\lambda), y=f(b)-f(\lambda a / b)$, and $z=f(a)-f(b)$. Using these new variables the claimed inequality (3.1) is equivalent to

$$
f(\lambda)(z-x)-x(x+y) \leq 0 .
$$

The $z \leq x$ case is clear (since $x \geq 0$ and $x+y>0$ ) while the case $z>x$ is actually vacuous. Indeed, $z>x$, i.e. $f(a)-f(b)>f(\lambda a / b)-f(\lambda)$, would be equivalent to

$$
\frac{f(a)-f(\lambda a / b)}{\lambda-b}>\frac{f(b)-f(\lambda)}{\lambda-b}
$$

which again is equivalent to

$$
\frac{a}{b} \cdot \frac{f\left(\frac{\lambda a}{b}\right)-f(a)}{\frac{\lambda a}{b}-a}<\frac{f(\lambda)-f(b)}{\lambda-b} .
$$

Recall that $a<\lambda a / b \leq b<\lambda$. By two applications of the mean value theorem there exist $c \in(a, \lambda a / b)$ and $d \in(b, \lambda)$ (in particular, $c<d$ must hold), such that

$$
f^{\prime}(c)=\frac{f\left(\frac{\lambda a}{b}\right)-f(a)}{\frac{\lambda a}{b}-a} \quad \text { and } \quad f^{\prime}(d)=\frac{f(\lambda)-f(b)}{\lambda-b} .
$$

Since, by assumption, the first derivatives of $f$ are negative in $(\delta, \lambda)$, we obtain from (3.2) the following string of inequalities:

$$
\frac{a}{b} f^{\prime}(c)<f^{\prime}(d)<0
$$

If we substract $f^{\prime}(c)$ from both sides of the first inequality, and subsequently divide by $d-c$, we obtain

$$
\left(\frac{a}{b}-1\right) \frac{f^{\prime}(c)}{d-c}<\frac{f^{\prime}(d)-f^{\prime}(c)}{d-c}
$$

Now since $\frac{a}{b}-1<0$ and $f^{\prime}(c)<0$ (by assumption), the product on the left-hand side of the inequality is positive. But the expression on the right-hand side is negative, as by the mean value theorem it is equal to the second derivative of $f$ at some point in $(c, d)$, which by assumption must be negative. This is a contradiction, thus the lemma is proved.

Now we are ready to prove Theorem 8 . 
Proof of Theorem [8. By the remarks following Definition 2 it suffices to show the continuous log-concavity of the $a, b ; q$-numbers (1.1), i.e.,

$$
\frac{[x+r]_{a, b ; q}[x-r]_{a, b ; q}}{[x]_{a, b ; q}^{2}} \leq 1 .
$$

Using the definition of the $a, b ; q$-numbers (1.1), after some cancellations, we see that the left-hand side of (3.3) is

$$
\begin{aligned}
& \frac{\left(1-q^{x+r}\right)\left(1-q^{x-r}\right)}{\left(1-q^{x}\right)^{2}} \frac{\left(1-a q^{x+r}\right)\left(1-a q^{x-r}\right)}{\left(1-a q^{x}\right)^{2}} \\
& \times \frac{\left(1-b q^{x}\right)^{2}}{\left(1-b q^{x+r}\right)\left(1-b q^{x-r}\right)} \frac{\left(1-a q^{x} / b\right)^{2}}{\left(1-a q^{x+r} / b\right)\left(1-a q^{x-r} / b\right)} .
\end{aligned}
$$

For a fixed pair of real numbers $x \geq r>0$, let

$$
f(u):=f_{q, x, r}(u)=\frac{\left(1-u q^{x+r}\right)\left(1-u q^{x-r}\right)}{\left(1-u q^{x}\right)^{2}} \quad \text { for } u \in[0,1] .
$$

We have

and

$$
f^{\prime}(u)=-\frac{\left(1-q^{r}\right)^{2}\left(1+u q^{x}\right)}{\left(1-u q^{x}\right)^{3}} q^{x-r}<0
$$

$$
f^{\prime \prime}(u)=-\frac{2\left(1-q^{r}\right)^{2}\left(2+u q^{x}\right)}{\left(1-u q^{x}\right)^{4}} q^{2 x-r}<0,
$$

for any $u \in(0,1)$, which allows us to apply Lemma 9 with $f$ as defined in (3.5), with $\delta=0$ and $\lambda=1$. This immediately establishes that the expression in (3.4) is $\leq 1$.

\section{4. $a, b ; q$-BINOMIAL COEFFICIENTS AND LOG-CONCAVITY}

In this section, we recall the definition of the $a, b ; q$-analogue of binomial coefficients (that first appeared in work of the first author [9] in the context of enumeration of weighted lattice paths and subsequently were studied in further work of the first author [10] in connection with a non-commutative binomial theorem, and in joint work of the first author with Yoo [11, 12]; in particular, these papers contain their recurrence relations and combinatorial interpretations, also for the more general $a, b ; q, p$ - or elliptic binomial coefficients, which include the $a, b ; q$-binomial coefficients obtained by letting $p \rightarrow 0$ ).

For the following definition we restrict the base $q$ to satisfy $0<|q|<1$ (while for studying inequalities we further assume $q$ to be real and satisfy $0<q<1$ ). For parameter $a \in \mathbb{C}$ and lower index $k \in \mathbb{C}$, the $q$-shifted factorial is defined as

$$
(a ; q)_{k}:=\frac{(a ; q)_{\infty}}{\left(a q^{k} ; q\right)_{\infty}}, \quad \text { where } \quad(a ; q)_{\infty}=\prod_{j \geq 0}\left(1-a q^{j}\right)
$$

cf. [3]. (For $a=q^{-n}$ with integers $n \geq k \geq 0$ the above definition for $(a ; q)_{k}$ involves a pole which however can be removed.) 
For brevity, we use the following compact notation for products of $q$-shifted factorials:

$$
\left(a_{1}, \ldots, a_{m} ; q\right)_{k}:=\left(a_{1} ; q\right)_{k} \cdots\left(a_{m} ; q\right)_{k}, \quad \text { where } \quad k \in \mathbb{C} \cup\{\infty\} .
$$

For $x, k, a, b, q \in \mathbb{C}, 0<|q|<1$, we define the $a, b ; q$-binomial coefficient as

$$
\begin{aligned}
{\left[\begin{array}{l}
x \\
k
\end{array}\right]_{a, b ; q}: } & =\frac{\left(q^{1+k}, a q^{1+k}, b q^{1+k}, a q^{1-k} / b ; q\right)_{x-k}}{\left(q, a q, b q^{1+2 k}, a q / b ; q\right)_{x-k}} \\
& =\frac{\left(q^{1+x-k}, a q^{1+x-k}, b q^{1+k}, a q^{1-k} / b ; q\right)_{k}}{\left(q, a q, b q^{1+x}, a q^{1+x-2 k} / b ; q\right)_{k}} .
\end{aligned}
$$

(Note that the $a, b ; q$-binomial coefficients are not symmetric in $k$ and $x-k$, contrary to the symmetry for the ordinary and basic binomial coefficients.) If we assume $x$ and $k$ to be non-negative integers, and replace $b$ by $b q$ on the right-hand side, then definition (4.1) matches that of the $a, b$; $q$-binomial coefficient as used in [9, 10, 11, 12, 13, 15].

If we let $k=1$ and divide $b$ by $q$, the $a, b ; q$-binomial coefficient reduces to the $a, b ; q$ number defined in (1.1):

$$
\left[\begin{array}{c}
x \\
1
\end{array}\right]_{a, b / q ; q}=[x]_{a, b ; q}
$$

We define the $a ; q$ and $(b ; q)$ analogues of binomials by taking limits $b \rightarrow \infty$ and $a \rightarrow 0$ of (4.1), respectively. Then

$$
\left[\begin{array}{l}
x \\
k
\end{array}\right]_{a ; q}:=\frac{\left(q^{1+k}, a q^{1+k} ; q\right)_{x-k}}{(q, a q ; q)_{x-k}} q^{k(k-x)}, \quad \text { and } \quad\left[\begin{array}{l}
x \\
k
\end{array}\right]_{(b ; q)}:=\frac{\left(q^{1+k}, b q^{1+k} ; q\right)_{x-k}}{\left(q, b q^{1+2 k} ; q\right)_{x-k}}
$$

(While the $a$; $q$-binomial coefficients are symmetric in $k$ and $x-k$, the $(b ; q)$-binomial coefficients are not.) It is clear that

$$
\left[\begin{array}{l}
x \\
1
\end{array}\right]_{a ; q}=[x]_{a ; q} \quad \text { and } \quad\left[\begin{array}{l}
x \\
1
\end{array}\right]_{(b / q ; q)}=[x]_{(b ; q)},
$$

with the $a ; q$ - and $(b ; q)$-numbers defined in (2.4) and (2.5), respectively.

Parallel to Section 3, we start our log-concavity discussion with the $a$; $q$-binomial coefficients. For these we have continuous strong log-concavity with respect to the lower parameter of the binomial coefficient.

Theorem 10. For any real $a, x, y, k, l, r, q$ satisfying $0<q<1,0 \leq a<1, x \geq y$, $k \geq l \geq r \geq 0$, and $y-l \geq x-k$, we have the continuous strong log-concavity

$$
\left[\begin{array}{l}
x \\
k
\end{array}\right]_{a ; q}\left[\begin{array}{l}
y \\
l
\end{array}\right]_{a ; q} \geq\left[\begin{array}{c}
x \\
k+r
\end{array}\right]_{a ; q}\left[\begin{array}{c}
y \\
l-r
\end{array}\right]_{a ; q} .
$$


Proof. After cancellations, we see that

$$
\begin{aligned}
\frac{\left[\begin{array}{c}
x \\
k+r
\end{array}\right]_{a ; q}\left[\begin{array}{c}
y \\
l-r
\end{array}\right]_{a ; q}}{\left[\begin{array}{l}
x \\
k
\end{array}\right]_{a ; q}\left[\begin{array}{c}
y \\
l
\end{array}\right]_{a ; q}}= & q^{2 r(r+k-l)} \frac{\left(q^{1+x-k-r}, a q^{1+x-k-r}, q^{1+k+r}, a q^{1+k+r} ; q\right)_{\infty}}{\left(q^{1+x-k}, a q^{1+x-k}, q^{1+k}, a q^{1+k} ; q\right)_{\infty}} \\
& \times \frac{\left(q^{1+y-l+r}, a q^{1+y-l+r}, q^{1+l-r}, a q^{1+l-r} ; q\right)_{\infty}}{\left(q^{1+y-l}, a q^{1+y-l}, q^{1+l}, a q^{1+l} ; q\right)_{\infty}} .
\end{aligned}
$$

Now, $q^{2 r(r+l-l)} \leq 1$ and by taking different instances of Proposition 4, we have

$$
\frac{\left(1-\nu q^{k+r}\right)\left(1-\nu q^{l-r}\right)}{\left(1-\nu q^{k}\right)\left(1-\nu q^{l}\right)} \leq 1, \quad \text { and } \quad \frac{\left(1-\nu q^{y-l+r}\right)\left(1-\nu q^{x-k-r}\right)}{\left(1-\nu q^{y-l}\right)\left(1-\nu q^{x-k}\right)} \leq 1,
$$

for $\nu=q^{j}$ and $\nu=a q^{j}$, where $j=1,2, \ldots$ Thus the infinite product is also $\leq 1$ and the theorem follows.

We note that rhe $(b ; q)$-binomial coefficients and $a, b ; q$-binomial coefficients do not appear to be log-concave with respect to their lower parameter.

We now turn back to the $a, b ; q$-binomial coefficients. We are able to prove the discrete strong log-concavity of $\left[\begin{array}{l}x \\ k\end{array}\right]_{a, b ; q}$ (here $x$ may be real but $k$ should be a non-negative integer) with respect to to the upper parameter $x$ but require that the range of the variables $a, b$ depends on the lower parameter $k$.

Theorem 11. For any real $a, b, x, y, q$ and non-negative integer $k$ satisfying $0<q<1$, $0<a \leq b q^{k}<1$, and $x \geq y \geq 1$, with the difference $x-y$ being a non-negative integer, we have the discrete strong log-concavity

$$
\left[\begin{array}{l}
x \\
k
\end{array}\right]_{a, b ; q}\left[\begin{array}{l}
y \\
k
\end{array}\right]_{a, b ; q} \geq\left[\begin{array}{c}
x+1 \\
k
\end{array}\right]_{a, b ; q}\left[\begin{array}{c}
y-1 \\
k
\end{array}\right]_{a, b ; q} .
$$

Proof. All we need to show is that

$$
\frac{\left[\begin{array}{c}
x+1 \\
k
\end{array}\right]_{a, b ; q}\left[\begin{array}{c}
x-1 \\
k
\end{array}\right]_{a, b ; q}}{\left[\begin{array}{l}
x \\
k
\end{array}\right]_{a, b ; q}^{2}} \leq 1
$$

as then the iteration of the inequality

$$
\frac{\left[\begin{array}{c}
x \\
k
\end{array}\right]_{a, b ; q}}{\left[\begin{array}{c}
x+1 \\
k
\end{array}\right]_{a, b ; q}} \geq \frac{\left[\begin{array}{c}
x-1 \\
k
\end{array}\right]_{a, b ; q}}{\left[\begin{array}{l}
x \\
k
\end{array}\right]_{a, b ; q}}
$$

leads to (4.5). After canceling common factors, we obtain

where

$$
\frac{\left[\begin{array}{c}
x+1 \\
k
\end{array}\right]_{a, b ; q}\left[\begin{array}{c}
x-1 \\
k
\end{array}\right]_{a, b ; q}}{\left[\begin{array}{l}
x \\
k
\end{array}\right]_{a, b ; q}^{2}}=\frac{g(1) g(a)}{g\left(b q^{k}\right) g\left(a / b q^{k}\right)}
$$

$$
g(u):=g_{q, x, k}(x)=\frac{\left(1-u q^{x-k}\right)\left(1-u q^{x+1}\right)}{\left(1-u q^{x-k+1}\right)\left(1-u q^{x}\right)} .
$$


Observe that

$$
g_{q, x, k}(u)=\prod_{i=1}^{k} f_{q, x-k+i, 1}(u),
$$

where $f_{q, x, 1}(u)$ is as defined in (3.5). Therefore, the right-side of (4.6) can be rewritten as

$$
\frac{\left[\begin{array}{c}
x+1 \\
k
\end{array}\right]_{a, b ; q}\left[\begin{array}{l}
x-1 \\
k-1
\end{array}\right]_{a, b ; q}}{\left[\begin{array}{l}
x \\
k
\end{array}\right]_{a, b ; q}^{2}}=\prod_{j=1}^{k} \frac{f_{q, x-k+j, 1}(1) f_{q, x-k+j, 1}(a)}{f_{q, x-k+j, 1}\left(b q^{k}\right) f_{q, x-k+j, 1}\left(a / b q^{k}\right)} .
$$

The $k$ terms of the product are each less than or equal to 1 by the $\delta=0, \lambda=1$ and $b \mapsto b q^{k}$ case of Lemma 3.1 with $f(u)$ as defined in (3.5) but with $b$ replaced by $b q^{k}$.

This is consistent with the log-concavity of the numbers $[n]_{a, b ; q}$ that we proved in Theorem 8, that is namely just the $k=1$ and $b \mapsto b / q$ case of Theorem 11 .

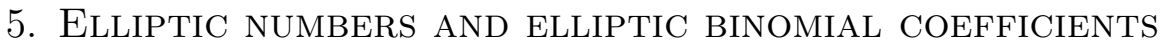

We start with explaining some standard notions about theta functions and elliptic functions. A function $g$ of a complex variable $z$ is elliptic if it is meromorphic and doubly periodic. It follows from the theory of abelian functions (cf. [16]) that there exists a non-negative integer $s$ and complex numbers $a_{1}, \ldots a_{s}, b_{1}, \ldots, b_{s}, c$ and $p$ with $0<|p|<1$ such that

$$
g(z)=c \frac{\theta\left(a_{1} z, \ldots, a_{s} z ; p\right)}{\theta\left(b_{1} z, \ldots, b_{s} z ; p\right)}, \quad \text { where } \quad a_{1} \cdots a_{s}=b_{1} \cdots b_{s} \neq 0 .
$$

Here, $\theta\left(x_{1}, \ldots, x_{s} ; p\right):=\theta\left(x_{1} ; p\right) \cdots \theta\left(x_{s} ; p\right)$, where, for $x \neq 0$ and $|p|<1$,

$$
\theta(x ; p)=(x, p / x ; p)_{\infty}
$$

is the modified Jacobi theta function (which in short we refer to as theta function) of argument $x$ and nome $p$. It is clear that for $p=0$ we have $\theta(x ; 0)=(1-x)$. So the theta function can be considered to be a $p$-extension of a linear factor. The following properties hold for the theta function:

$$
\begin{aligned}
\theta(x ; p) & =-x \theta(1 / x ; p), \\
\theta(p x ; p) & =-\frac{1}{x} \theta(x ; p),
\end{aligned}
$$

and the addition formula

$$
\theta(x y, x / y, u t, u / t ; p)-\theta(x t, x / t, u y, u / y ; p)=\frac{u}{y} \theta(y t, y / t, x u, x / u ; p) .
$$

The two identities in (5.1), which can be referred to as inversion formula and quasiperiodicity, are readily shown from the definition of the theta function, while the theta function addition formula (originally due to Weierstraß, see [17, p. 451, Example 5]) in (5.2) is not obvious but crucial in the theory of elliptic hypergeometric functions. (See 
e.g. Rosengren's lecture notes [6, Sec. 1.4] for the standard derivation of (5.2) involving complex analysis.)

We recall further classical facts about theta functions which we need. In particular, we would like to have formulas involving their first and second derivatives, so we can apply Lemma 9 with $f$ involving a quotient of theta functions, rather than a quotient of linear factors as in (3.5). This will enable us to extend Theorems 8 and 11 to the elliptic setting.

The modified Jacobi theta function is a special case of a sigma function. According to [17, p. 473, §21.43] the function $\sigma(z)=\sigma\left(z \mid \omega_{1}, \omega_{2}\right)$ formed with the two periods $2 \omega_{1}, 2 \omega_{2}$, is expressible in the form

$$
\begin{aligned}
\sigma\left(z \mid \omega_{1}, \omega_{2}\right) & =\frac{2 \omega_{1}}{\pi} \exp \left(\frac{\eta z^{2}}{2 \omega_{1}}\right) \sin \left(\frac{\pi z}{2 \omega_{1}}\right) \prod_{n=1}^{\infty} \frac{\left(1-2 \tilde{p}^{2 n} \cos \frac{\pi z}{\omega_{1}}+\tilde{p}^{4 n}\right)}{\left(1-\tilde{p}^{2 n}\right)^{2}} \\
& =\omega_{1} e^{\eta z^{2} / 2 \omega_{1}}\left(e^{\pi \mathrm{i} / 2 \omega_{1}}-e^{-\pi \mathrm{i} / 2 \omega_{1}}\right) \frac{\left(\tilde{p}^{2} e^{\pi \mathrm{i} z / \omega_{1}}, \tilde{p}^{2} e^{-\pi \mathrm{i} z / \omega_{1}} ; \tilde{p}^{2}\right)_{\infty}}{\pi \mathrm{i}\left(\tilde{p}^{2} ; \tilde{p}^{2}\right)_{\infty}^{2}} \\
& =\frac{\mathrm{i} \omega_{1} e^{\left(\eta z^{2}-\pi \mathrm{i}\right) / 2 \omega_{1}} \theta\left(e^{\pi \mathrm{i} / 2 \omega_{1}} ; \tilde{p}^{2}\right)}{\pi\left(\tilde{p}^{2} ; \tilde{p}^{2}\right)_{\infty}^{2}}
\end{aligned}
$$

where

$$
\eta=\frac{\pi^{2}}{12 \omega_{1}}\left(1-24 \sum_{n=1}^{\infty} \frac{\tilde{p}^{2 n}}{\left(1-\tilde{p}^{2 n}\right)^{2}}\right) \quad \text { and } \quad \tilde{p}=e^{\frac{\pi \mathrm{i} \omega_{2}}{\omega_{1}}} .
$$

From (5.2) it is immediate that the sigma function satisfies the addition formula (cf. [17, p. 451, Ex. 5])

$$
\begin{aligned}
\sigma(x+y) \sigma(x-y) \sigma(u+t) \sigma(u-t) & -\sigma(x+t) \sigma(x-t) \sigma(u+y) \sigma(u-y) \\
& =\sigma(y+t) \sigma(y-t) \sigma(x+u) \sigma(x-u) .
\end{aligned}
$$

Now let $\zeta(z):=\frac{\mathrm{d}}{\mathrm{d} z} \log \sigma(z)$ and $\wp(z):=-\frac{\mathrm{d}}{\mathrm{d} z} \zeta(z)$ (called the Weierstra $\beta \zeta$-function and Weierstraß $\wp$-function, respectively; the latter is actually an elliptic function). Differentiation of both sides of (5.4) with respect to $x$, followed by division by $\sigma(x+y) \sigma(x-$ $y) \sigma(u+t) \sigma(u-t)$ and putting $u=x$, gives (cf. [17, p. 461, Ex. 38])

$$
\zeta(x+y)+\zeta(x-y)-\zeta(x+t)-\zeta(x-t)=\frac{\sigma^{\prime}(0) \sigma(2 x) \sigma(y+t) \sigma(y-t)}{\sigma(x+y) \sigma(x-y) \sigma(x+t) \sigma(x-t)} .
$$

Now it is not difficult to show directly, using (5.3), that $\sigma^{\prime}(0)=1$. After making the simultaneous substitutions $(x, y, t) \mapsto((u+v) / 2,(u-v) / 2,-w-(u+v) / 2)$ in (5.5) we obtain

$$
\zeta(u)+\zeta(v)+\zeta(w)-\zeta(u+v+w)=\frac{\sigma(u+v) \sigma(u+w) \sigma(v+w)}{\sigma(u) \sigma(v) \sigma(w) \sigma(u+v+w)}
$$

which after the simultaneous substitutions $(u, v, w) \mapsto(2 u, 2 v,-u-v)$ reduces to

$$
\zeta(2 u)+\zeta(2 v)-2 \zeta(u+v)=\frac{\sigma(2 u+2 v) \sigma^{2}(u-v)}{\sigma(2 u) \sigma(2 v) \sigma^{2}(u+v)} .
$$


Finally, by differentiating (5.6) with respect to $x$ and applying (5.6) to the result, one obtains, after another substitution of variables, (cf. [17, p. 451, Ex. 1])

$$
\wp(v)-\wp(u)=\frac{\sigma(u-v) \sigma(u+v)}{\sigma^{2}(u) \sigma^{2}(v)} .
$$

This important formula can be used to prove the addition formula in (5.4) without knowing the definition of the Weierstraß $\wp$-function. While we will actually not use this function and the formula in (5.8) in this paper, we nevertheless include this very classical material to make our survey in this section more complete.

In the following we will choose as half-periods $\omega_{1}=\frac{1}{2}, \omega_{2}=\frac{\tau}{2}$ (with $\tau \in \mathrm{i} \mathbb{R}^{+}$), and put $p=\tilde{p}^{2}$, so $p=e^{2 \pi \mathrm{i} \tau}$. (Notice that the condition on $\tau$ guarantees that $0<|p|<1$.) With these specializations we have

$$
\sigma(z)=\sigma\left(z \mid \frac{1}{2}, \frac{\tau}{2}\right)=\frac{\mathrm{i} e^{\eta z^{2}-\pi \mathrm{i} z} \theta\left(e^{2 \pi \mathrm{i} z} ; p\right)}{2 \pi(p ; p)_{\infty}^{2}}
$$

where

$$
\eta=\frac{\pi^{2}}{6}\left(1-24 \sum_{n=1}^{\infty} \frac{p^{n}}{\left(1-p^{n}\right)^{2}}\right) .
$$

We are now ready to define our elliptic numbers and elliptic binomial coefficients. For $a, b, q, p \in \mathbb{C}$ with $|p|<1$ we define the elliptic (or $a, b ; q, p$-)extension of a number $x$ (which does not need to be an integer) as follows:

$$
[x]_{a, b ; q, p}=\frac{\theta\left(q^{x}, a q^{x}, b q, a q / b ; p\right)}{\theta\left(q, a q, b q^{x}, a q^{x} / b ; p\right)} .
$$

(This definition corresponds to that for the elliptic numbers considered in [11, 12, 13, 14] subject to the substitution $b \mapsto b q^{-1}$.)

From (5.2) we have for all $x$ and $y$ the following addition formula for the elliptic numbers:

$$
[x]_{a, b ; q, p}+W_{a, b ; q, p}(x)[y-x]_{a q^{2 x}, b q^{x} ; q, p}=[y]_{a, b ; q, p},
$$

where $W_{a, b ; q, p}(x)$ is the elliptic-weight, defined by

$$
W_{a, b ; q, p}(x)=\frac{\theta\left(a q^{1+2 x}, b, b q, a / b, a q / b ; p\right)}{\theta\left(a q, b q^{x}, b q^{1+x}, a q^{x} / b, a q^{1+x} / b ; p\right)} q^{x} .
$$

It is clear that the properties (2.2) and (2.3) readily extend to the elliptic level.

For parameter $a \in \mathbb{C}$, base $0<|q|<1$, nome $|p|<1$ and non-negative integer $k$, the theta (or $q, p$-) shifted factorial is defined as

$$
(a ; q, p)_{k}:=\theta\left(a, a q, \ldots, a q^{k-1} ; p\right),
$$

cf. [3, Ch. 11]. For brevity, we use the following compact notation for products of theta shifted factorials:

$$
\left(a_{1}, \ldots, a_{m} ; q, p\right)_{k}:=\left(a_{1} ; q, p\right)_{k} \cdots\left(a_{m} ; q, p\right)_{k}, \quad \text { where } \quad k \in \mathbb{N}_{0} .
$$


Further, for $x, a, b, q, p \in \mathbb{C}$ with $0<|q|<1,|p|<1$, and a non-negative integer $k$ we define the elliptic (or $a, b ; q, p$-) binomial coefficient as

$$
\left[\begin{array}{l}
x \\
k
\end{array}\right]_{a, b ; q, p}:=\frac{\left(q^{1+x-k}, a q^{1+x-k}, b q^{1+k}, a q^{1-k} / b ; q, p\right)_{k}}{\left(q, a q, b q^{1+x}, a q^{1+x-2 k} / b ; q, p\right)_{k}}
$$

If we assume $x$ and $k$ being nonnegative integers, and replace $b$ by $b q$ on the right-hand side, the definition in (5.12) matches that of the elliptic binomial coefficient as used in [9, 11, 12]. Clearly, $\left[\begin{array}{l}x \\ 1\end{array}\right]_{a, b / q ; q, p}=[x]_{a, b ; q, p}$.

\section{LOG-CONCAVITY OF ELLIPTIC NUMBERS AND ELLIPTIC BINOMIAL COEFFICIENTS}

Just as the $a, b ; q$-numbers satisfy an $a, b ; q$-version of continuous strong log-concavity where certain shifts of the variables $a$ and $b$ occur (see Theorem 7), the elliptic numbers satisfy the following result:

Theorem 12. Let $q, p, x, y, r, a, b$ be real numbers satisfying $0<q<1,0<p<1$, $x \geq y \geq r \geq 0$, and $0<a<b<1$. Then the elliptic numbers satisfy the inequality

$$
[x]_{a q^{2 r}, b q^{r} ; q, p}[y]_{a, b ; q, p} \geq[x+r]_{a, b ; q, p}[y-r]_{a q^{2 r}, b q^{r} ; q, p} .
$$

Proof. The proof is just like that of Theorem 7 , but we use (5.11) instead of (2.1).

In the remaining section we will consider log-concavity results where the variables $a$ and $b$ do not shift. Our results crucially depend on the following proposition.

Proposition 13. Let $q, p, x, r$ be real numbers satisfying $0<q<1, x \geq r>0$ and $0<p<q^{2 r}$, and let the function $f$ be defined by

$$
f(u):=f_{q, p, x, r}(u)=\frac{\theta\left(u q^{x+r}, u q^{x-r} ; p\right)}{\theta^{2}\left(u q^{x} ; p\right)} \quad \text { for } u \in[\delta, \lambda],
$$

where $\delta=p q^{-x-r}$ and $\lambda=q^{r-x}$. Then $f$ is continuous and positive on the interval $[\delta, \lambda]$, with first and second derivatives being negative on the interval $(\delta, \lambda)$.

Proof. The continuity and positivity is clear. To compute the derivatives on $(\delta, \lambda)$, we rewrite $f(u)$ in terms of the sigma function. Let $p=e^{2 \pi \mathrm{i} \tau}, q=e^{2 \pi \mathrm{i} \nu}$, and $u=e^{2 \pi \mathrm{i} z}$, where $\tau, \nu, z \in \mathrm{i} \mathbb{R}$. We have $\mathrm{d} u=2 \pi \mathrm{i} e^{2 \pi \mathrm{i} z} \mathrm{~d} z$, or equivalently, $\frac{\mathrm{d}}{\mathrm{d} z}=2 \pi \mathrm{i} u \frac{\mathrm{d}}{\mathrm{d} u}$, and, according to (5.9a),

$$
f\left(e^{2 \pi \mathrm{i} z}\right)=\frac{\theta\left(e^{2 \pi \mathrm{i}((x+r) \nu+z)}, e^{2 \pi \mathrm{i}((x-r) \nu+z)} ; p\right)}{\theta^{2}\left(e^{2 \pi \mathrm{i}(x \nu+z)} ; p\right)}=e^{-2 \eta r^{2} \nu^{2}} \frac{\sigma(z+(x+r) \nu) \sigma(z+(x-r) \nu)}{\sigma^{2}(z+x \nu)} .
$$

Thus, the first derivative of $f$ is

$$
\frac{\mathrm{d}}{\mathrm{d} u} f(u)=\frac{e^{-2 \pi \mathrm{i} z}}{2 \pi \mathrm{i}} \frac{\mathrm{d}}{\mathrm{d} z} f\left(e^{2 \pi \mathrm{i} z}\right)=\frac{e^{-2 \pi \mathrm{i} z-2 \eta r^{2} \nu^{2}}}{2 \pi \mathrm{i}} \frac{\mathrm{d}}{\mathrm{d} z} \frac{\sigma(z+(x+r) \nu) \sigma(z+(x-r) \nu)}{\sigma^{2}(z+x \nu)}
$$




$$
\begin{aligned}
\begin{aligned}
=\frac{e^{-2 \pi \mathrm{i} z-2 \eta r^{2} \nu^{2}}}{2 \pi \mathrm{i} \sigma^{3}(z+x \nu)}[ & \sigma(z+x \nu) \sigma(z+(x+r) \nu) \sigma^{\prime}(z+(x-r) \nu) \\
& +\sigma(z+x \nu) \sigma^{\prime}(z+(x+r) \nu) \sigma(z+(x-r) \nu) \\
& \left.-2 \sigma^{\prime}(z+x \nu) \sigma(z+(x+r) \nu) \sigma(z+(x-r) \nu)\right]
\end{aligned} \\
=\frac{e^{-2 \pi \mathrm{i} z}}{2 \pi \mathrm{i}} f\left(e^{2 \pi \mathrm{i} z}\right)[\zeta(z+(x+r) \nu)+\zeta(z+(x-r) \nu)-2 \zeta(z+x \nu)] \\
=\frac{e^{-2 \pi \mathrm{i} z-2 \eta r^{2} \nu^{2}}}{2 \pi \mathrm{i}} \frac{\sigma(2(z+x \nu)) \sigma^{2}(r \nu)}{\sigma^{4}(z+x \nu)} \\
=-q^{x-r} \frac{(p ; p)_{\infty}^{2} \theta^{2}\left(q^{r} ; p\right) \theta\left(u^{2} q^{2 x} ; p\right)}{\theta^{4}\left(u q^{x} ; p\right)}
\end{aligned}
$$

where we applied (5.7) in the penultimate equality, and used (5.9a) to rewrite the expression in terms of the modified theta function in the last equality. The final expression obtained for the first derivative of $f(u)$ is clearly negative. (The minus sign in front of the product stems from collecting all the factors i from the applications of (5.9a); indeed, $\mathrm{i}^{3} / \mathrm{i}^{5}=-1$.)

We still need to compute the second derivative of $f$ and show that it is also negative. We have

$$
\begin{aligned}
\frac{\mathrm{d}^{2}}{\mathrm{~d} u^{2}} f(u) & =-q^{x-r}(p ; p)_{\infty}^{2} \theta^{2}\left(q^{r} ; p\right) \frac{\mathrm{d}}{\mathrm{d} u} \frac{\theta\left(u^{2} q^{2 x} ; p\right)}{\theta^{4}\left(u q^{x} ; p\right)} \\
& =-q^{x-r} \frac{(p ; p)_{\infty}^{2} \theta^{2}\left(q^{r} ; p\right) \theta\left(u^{2} q^{2 x} ; p\right)}{\theta^{4}\left(u q^{x} ; p\right)}\left(\frac{\mathrm{d}}{\mathrm{d} u} \log \theta\left(u^{2} q^{2 x} ; p\right)-4 \frac{\mathrm{d}}{\mathrm{d} u} \log \theta\left(u q^{x} ; p\right)\right) .
\end{aligned}
$$

All we need to do is to show that

$$
\frac{\mathrm{d}}{\mathrm{d} u} \log \theta\left(u^{2} q^{2 x} ; p\right)>4 \frac{\mathrm{d}}{\mathrm{d} u} \log \theta\left(u q^{x} ; p\right) .
$$

Since the logarithmic derivative of a product is the sum of the logarithmic derivatives, we thus need to show

$$
\begin{aligned}
& \sum_{j=0}^{\infty} \frac{\mathrm{d}}{\mathrm{d} u} \log \left[\left(1-p^{j} u^{2} q^{2 x}\right)+\left(1-p^{j+1} u^{-2} q^{-2 x}\right)\right] \\
& >4 \sum_{j=0}^{\infty} \frac{\mathrm{d}}{\mathrm{d} u} \log \left[\left(1-p^{j} u q^{x}\right)+\left(1-p^{j+1} u^{-1} q^{-x}\right)\right] .
\end{aligned}
$$

The inequality actually holds term-wise, for each $j$. Indeed, comparison of the logarithmic derivatives for fixed $j$ amounts to

$$
\frac{-2 p^{j} u q^{2 x}}{1-p^{j} u^{2} q^{2 x}}+\frac{4 p^{j+1} u^{-3} q^{-2 x}}{1-p^{j+1} u^{-2} q^{-2 x}}>\frac{-4 p^{j} q^{x}}{1-p^{j} u q^{x}}+\frac{4 p^{j+1} u^{-2} q^{-x}}{1-p^{j+1} u^{-1} q^{-x}},
$$


which is easy to verify, as already the first summand on the left-hand side is greater than the first summand on the right-hand side, which analogously holds for the second summands on both sides.

Having set up all the ingredients, the results now immediately extend from the $a, b ; q, p$ case to the elliptic case.

Theorem 14. The elliptic numbers $[x]_{a, b ; q, p}$ are continuously strongly log-concave. In particular, for all real numbers $q, p, x, y, r, a, b$ satisfying $0<q<1,0<p<q^{2 r}, x \geq y \geq$ $r \geq 0$, and $p q^{-x-r}<a<b<1$ we have

$$
[x]_{a, b ; q, p}[y]_{a, b ; q, p} \geq[x+r]_{a, b ; q, p}[y-r]_{a, b ; q, p} .
$$

Proof. The proof follows the lines of that of the proof of Theorem 8 but we use, instead of (3.5), $f(u)$ as defined in (6.1), where $u \in[\delta, 1]$ with $\delta=p q^{-x-r}$. As a consequence of Proposition [13, $f$ satisfies on $[\delta, 1]$ (which is contained in $[\delta, \lambda]$ with $\lambda=q^{r-x}$ ) the necessary requirements for application of Lemma 9, which we invoke with $\lambda=1$.

Theorem 15. For any real $q, p, a, b, x, y$ and non-negative integer $k$ satisfying $0<q<1$, $0<p<q^{2}, p q^{-x-1}<a \leq b q^{k}<1$, and $x \geq y \geq 1$, with the difference $x-y$ being a nonnegative integer, we have the discrete strong log-concavity of elliptic binomial coefficients

$$
\left[\begin{array}{l}
x \\
k
\end{array}\right]_{a, b ; q, p}\left[\begin{array}{l}
y \\
k
\end{array}\right]_{a, b ; q, p} \geq\left[\begin{array}{c}
x+1 \\
k
\end{array}\right]_{a, b ; q, p}\left[\begin{array}{c}
y-1 \\
k
\end{array}\right]_{a, b ; q, p} .
$$

Proof. The proof follows the lines of that of the proof of Theorem 11 but we use, instead of (3.5), $f(u)$ as defined in (6.1), where $u \in[\delta, 1]$ with $\delta=p q^{-x-1}$, for application of Lemma 9 ,

\section{Conclusion}

We have shown that fundamental log-concavity results which are known to hold for (the $q$-)numbers and ( $q$-) binomial coefficients can be extended to the $a, b ; q$-case and even to the elliptic case. Further closely related questions remain open, for instance about the log-concavity of $a, b ; q$ - or elliptic extensions of those other sequences considered by Sagan in [7] or of the $a, b ; q$ - or elliptic rook numbers (considered by the first author and Yoo [12, 13]).

\section{ACKNOWLEDGEMENTS}

All three authors thank the FWF Austrian Science Fund for generous support. In particular, the first author was partially supported by grants F50-08 and P32305, the second author was fully supported by grants F50-08 and F50-10, and the third author was fully supported by grants F50-07, F50-09 and F50-11. 


\section{REFERENCES}

[1] G. E. Andrews, The Theory of Partitions, Cambridge University Press, Cambridge, 1998.

[2] L. M. Butler, The $q$-log concavity of $q$-binomial coefficients, J. Combin. Th. Ser. A 54(1) (1990) $54-63$.

[3] G. Gasper and M. Rahman, Basic Hypergeometric Series, second edition, Encyclopedia of Mathematics and Its Applications 96, Cambridge University Press, Cambridge, 2004.

[4] S. I. Kalmykov and D. B. Karp, Inequalities for series in $q$-shifted factorials and $q$-gamma functions, J. Math. Anal. Appl. 460(1) (2018) 332-351.

[5] C. Krattenthaler, On the $q$-log-concavity of Gaussian binomial coefficients, Monatshefte Math. 107 (1989) 333-339.

[6] H. Rosengren, Elliptic hypergeometric functions, in Lectures on Orthogonal Polynomials and Special Functions, H. S. Cohl and M. E. H. Ismail, eds., Cambridge University Press, to appear; arXiv: 1608.06161.

[7] B. E. Sagan, Inductive proofs of q-log concavity, Discrete Math. 99(1-3) (1992) 289-306.

[8] D. Salwinski, The continuous binomial coefficient: an elementary approach, Amer. Math. Monthly 125(3) (2018) 231-244.

[9] M. J. Schlosser, Elliptic enumeration of nonintersecting lattice paths, J. Combin. Theory Ser. A 114(3) (2007) 505-521.

[10] M. J. Schlosser, A noncommutative weight-dependent generalization of the binomial theorem, Sém. Lothar. Combin. B81j (2020), 24 pp.

[11] M. J. Schlosser, and M. Yoo, An elliptic extension of the general product formula for augmented rook boards, Europ. J. Combin. 58 (2016) 247-266.

[12] M. J. Schlosser, and M. Yoo, Elliptic rook and file numbers, Electron. J. Combin. 24(1) (2017) \#P1.31, 47 pp.

[13] M. J. Schlosser, and M. Yoo, Elliptic extensions of the alpha-parameter model and the rook model for matchings, Adv. Appl. Math. 84 (2017) 8-33.

[14] M. J. Schlosser, and M. Yoo, Basic hypergeometric summations from rook theory, in Analytic Number Theory, Modular Forms and q-Hypergeometric Series in Honor of Krishna Alladi's 60th birthday, University of Florida, Gainesville, March 2016 (G.E. Andrews and F. Garvan, eds.), Springer Proc. Math. Stat. 221 (2017) 677-692

[15] M. J. Schlosser, and M. Yoo, Weight-dependent commutation relations and combinatorial identities, Discrete Math. 341 (2018) 2308-2325.

[16] H. Weber, Elliptische Functionen und Algebraische Zahlen, Vieweg-Verlag, Braunschweig, 1897.

[17] E. T. Whittaker and G. N. Watson, A Course of Modern Analysis, 4th ed., Cambridge University Press, Cambridge, 1962.

Fakultät für Mathematik, Universität Wien, Oskar-Morgenstern-Platz 1, A-1090 ViENNA, AUSTRIA

E-mail address: michael.schlosser@univie.ac.at

Fakultät für Mathematik, Universität Wien, Oskar-Morgenstern-Platz 1, A-1090 ViENNA, Austria

E-mail address: koushik.bapan.19@gmail.com

Johann Radon Institute for Computational and Applied Mathematics, Austrian Academy of Sciences, Altenbergerstrasse 69, A-4040 Linz, Austria

E-mail address: akuncu@risc.jku.at 